\title{
3 Perioperative respiratorische Komplikationen
}

\author{
Martin Zoremba
}

Adipositas bedingt eine 4-fach höhere Inzidenz von respiratorischen Komplikationen. Diese können sich in einer temporären Desaturation bis hin zu einer manifesten respiratorischen Insuffizienz äußern. Eine exakte präoperative Risikostratifizierung ist nicht möglich. Zusätzliche Untersuchungen (Spirometrie) konnten das perioperative Risiko für respiratorische Komplikationen nicht reduzieren. Morbidität und Mortalität steigen insbesondere in Kombination mit Nikotinabusus bei einem BMI > 30 rapide an.

\footnotetext{
Neben dem BMI gelten in einem 6-Faktoren-Risikomodell

- Rauchen in den letzten 8 Wochen präoperativ,

- Abdominaleingriffe,

- Malignome,

- Alter > 60 Jahre (Komorbiditäten) und

- eingeschränkte kognitive Funktionen

als Risikoindizes für postoperative pulmonale Komplikationen.
}

Dabei ist die Reduktion der Vitalkapazität abhängig vom Schweregrad der Adipositas. Betroffen sind besonders Patienten mit vorwiegend abdomineller Adipositas. Insbesondere die mit Adipositas einhergehende nicht unerhebliche Ko-Morbidität erschwert eine genaue Risikoklassifizierung. Verschiedene Faktoren spielen hierbei eine wichtige Rolle. Bereits der Einfluss einer Prämedikation (üblicherweise mit einem Benzodiazepin) am Vorabend besitzt eine nicht unerhebliche Wirkung auf die Reduktion der nächtlichen Atemregulation. Dies hat bei Adipositas eine besondere Bedeutung, da die Prävalenz an unerkannter OSA (obstruktiver Schlaf-Apnoe) mit steigendem BMI deutlich erhöht ist. Adipöse Patienten haben ein im Vergleich zum normalgewichtigen Kollektiv 10-30-fach höheres Risiko für OSA. Dabei wird bei bis zu 90\% der Risikopatienten von einer unerkannten OSA ausgegangen. Neben der zentralen Atemregulationsstörung spielen die im Rahmen der Adipositas verringerten pharyngalen Diameter eine nicht unerhebliche Rolle. In Verbindung mit einem erhöhten Patientenalter und einer veränderten Metabolisierung führt dies durch die in der perioperativen Phase applizierten Pharmaka zu einem rascheren Kollaps der oberen Atemwege, was Hypoventilation und Desaturation begünstigt. Bisher existieren nur wenige kontrollierte Studien, die pulmonale Komplikationen in diesem Setting exakt evaluiert haben. Deren Ergebnisse sind jedoch eindeutig. Sowohl die absolute Anzahl an ernsthaften pulmonalen Komplikationen (24\% vs. 9\%) und ungeplanten Intensivaufenthalten (20\% vs. $6 \%$ ) als auch die Verweildauer in der Klink waren statistisch signifikant $(\mathrm{p}<0.001)$ unterschiedlich. Dies wird im Rahmen der anästhesiologischen Prämedikationsvisite anhand einer ASA-II-Klassifizierung bei Adipositas allerdings als nur „leicht erhöhtes Narkoserisiko" unzureichend abgebildet. 


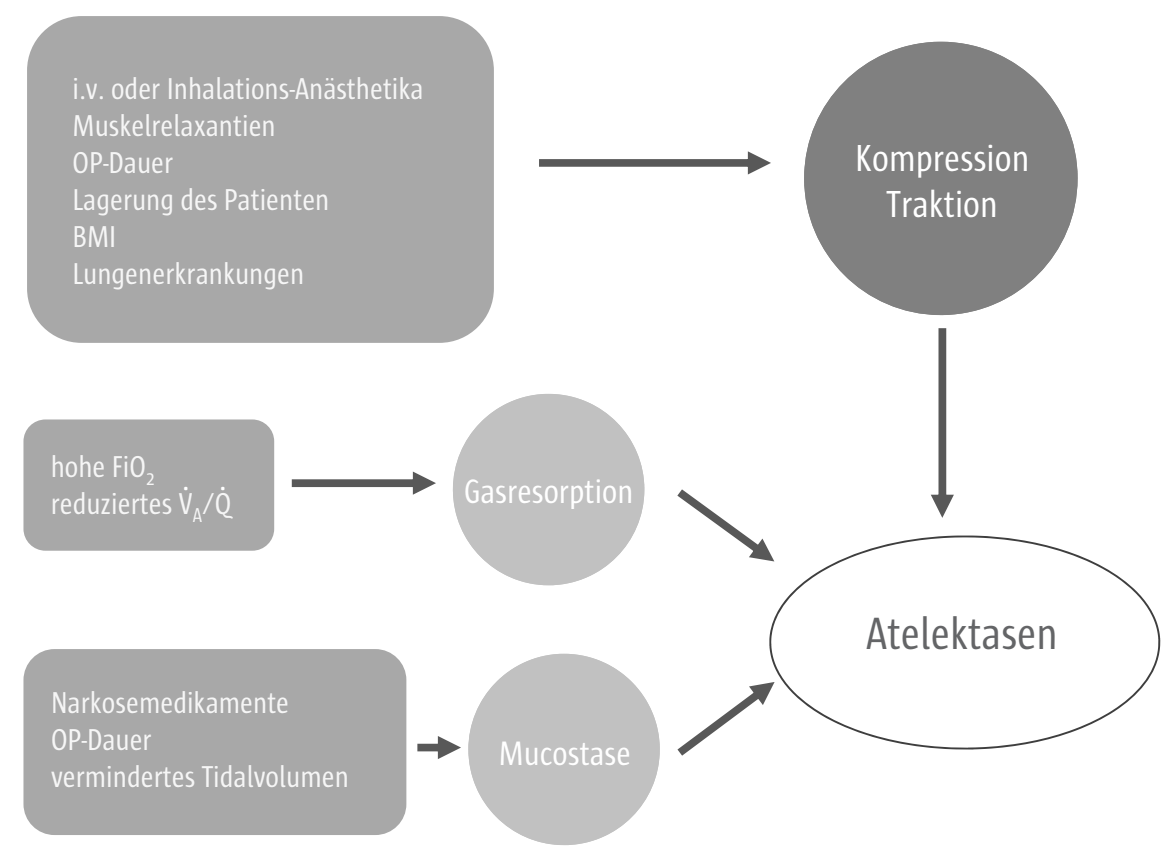

Abb. 2 Perioperative Einflussfaktoren auf die Ausprägung von Atelektasen (Duggan u. Kavanagh, Pulmonary Atelectasis. A Pathogenic Perioperative Entity, Anesthesiology 102, 838-854)

\section{Daher ist anzunehmen, dass die Adipositas, insbeson- dere bei OSA (unerkannt) eine größere Bedeutung für postoperative pulmonale Komplikationen besitzt als bislang vermutet.}

$\mathrm{Zu}$ den bereits präexistenten pathophysiologischen Veränderungen kommt es im Rahmen einer Allgemeinanästhesie mit maschineller Beatmung zu weitreichenden Veränderungen. Dies führt in der Summe zu einer vermehrten Atelektasenbildung und erhöhtem intrapulmonalem Shunt. In der perioperativen Phase sind vorrangig Resorptions/Kompressionsatelektasen vorhanden (s. Abb. 2). Diese sind bei über 90\% der erwachsenen Patienten bereits unmittelbar nach Narkoseinduktion im CT nachweisbar. Sie werden durch einen perioperativen Surfactantverlust der kollabierten Areale weiter begünstigt und können mehrere Tage persistieren. Kausal ist vor allem eine Reduktion der funktionellen Residualkapazität (FRC) mit konsekutivem alveolärem Kollaps (Atelektasen). Adipositas bzw. ein steigender BMI tragen zur Ausprägung dieser Atelektasen bei, was eine erhebliche Beeinträchtigung der pulmonalen Reserve bedeutet und respiratorische Komplikationen nach sich zieht.
Pathophysiologisch zeigt sich eine Verschlechterung der Lungencompliance. Der metabolische Bedarf der Atemmuskulatur steigt an und die Effizienz der Atemmuskulatur sinkt. Die wichtigsten mechanischen Faktoren sind die Abnahme der totalen respiratorischen systemischen Compliance sowie ein Anstieg der Atemwegsresistance, wodurch in der Summe die Atemarbeit um 30-70\% erhöht ist und eine raschere Erschöpfung der muskulären Atempumpe eintritt.

Infolge der veränderten Atemmechanik bzw. der Atelektasenbildung kommt es zu einer lokalen alveolären inflammatorischen Reaktion, welche eine Schädigung der alveolären Membran hervorruft und die Entstehung von Pneumonien begünstigt. Der alveoläre Kollaps zeigt sich postoperativ in einer restriktiven Ventilationsstörung mit einer nachhaltigen Reduktion der Lungenfunktionsparameter. Anhand der Blutgasanalyse lässt sich durch einen reduzierten Oxigenations-Index (OI, Horowitz Quotienten; $\mathrm{PaO}_{2} l$ $\mathrm{FiO}_{2}$ ) das Ausmaß der Atelektasen und der damit verbundenen pulmonalen Shuntfraktion abschätzen (Nunn 1989). Der pulmonale Shunt bzw. die HPV (hypoxisch-pulmonale Vasokonstriktion) bedingen zudem eine Erhöhung des pulmonal-arteriellen Druckes. Dies führt zu einer Rechtsherzbelastung und kann im Ext- 
remfall eine kardiale Dekompensation des rechtsventrikulären Stromgebietes zur Folge haben. Getriggert wird dieser Effekt zum einen durch eine (lokale) Hypoxie und zum anderen durch eine (lokale) Hyperkapnie (Azidose).

Aus diesem Grunde sollten auch ausgeprägte hyperkapnische Phasen mit begleitender respiratorischer Azidose bei kardiopulmonalen Risikopatienten unbedingt vermieden werden.

\section{Literatur}

Berg H, Roed |, Viby-Mogensen |, Mortensen CR, Engbäk |, Skovgaard

LT, Krintel || (1997) Residual neuromuscular block is a risk fac- tor for post operative pulmonary complications. A prospective, randomised, and blinded study of post operative pulmonary complications after atracurium, vecuronium and pancuronium. Acta Anaesthesiol Scand 41, 1095-1103

Brooks-Brunn JA (1997) Predictors of postoperative pulmonary complications following abdominal surgery. Chest $111,564-$ 571

Duggan M, Kavanagh BP (2005) Pulmonary Atelectasis. A Pathogenic Perioperative Entity. Anesthesiology 102, 838-854

Lawrence VA, Dhanda R, Hilsenbeck SG, Page CP (1996) Risk of pulmonary complications after elective abdominal surgery. Chest 110, 744-750

Nunn JF (1989) Applied Respiratory Physiology, ed. 3 Butterworths London

Ungern-Sternberg BS, Regli A, Reber A and Schneider MC (2005) Comparison of perioperative spirometric data following spinal or general anaesthesia in normal weight and overweight gynecological patients. Acta Anaesthesiol Scand 49, 940-948

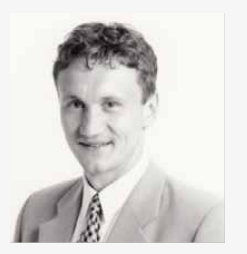

PD Dr. med. Martin Zoremba, D.E.A.A.

Nach Beendigung des Medizinstudiums an der Philipps Universität Marburg absolvierte er sein AIP an der Universität Gießen und ist seit 2000 in der Abteilung für Anästhesie und Intensivtherapie an der Universität Marburg tätig. Sein Forschungsschwerpunkt ist das perioperative Management von adipösen Patienten. Ein wichtiger Aspekt ist die Evaluation von „Fast-tracking-Prozeduren“ für Risikopatienten mit besonderem Fokus auf die perioperative respiratorische Funktion sowie postoperative schlafbezogene Atemstörungen. 